\title{
G-20: ESTRUCTURA Y ADECUACIÓN A SUS FINALIDADES EN EL MARCO ORGANIZATIVO E INSTITUCIONAL INTERNACIONAL ${ }^{1}$
}

\author{
Enrique Belda Pérez-Pedrero
}

doi: 10.18543/ed-64(1)-2016pp207-226

\begin{abstract}
SUMARIO: 1. El G-20 PARA EL DERECHO Y PARA LA ECONOMÍA. INTRODUCCIÓN JURÍDICA A UNA «INSTITUCIÓN» INSTRUMENTAL. 2. ¿LA ORGANIZACIÓN DEL G-20 POSIBILITA SUS FINES INSTITUCIONALES? CONGRUENCIA CON SUS FUNCIONES. 3. LA TEMÁTICA ECONÓMICA COMO EJE DE LA EXISTENCIA DEL G-20. LA PRESENCIA DEL DERECHO EN LA ECONOMÍA. ¿Y DE LA ECONOMÍA EN EL DERECHO? 4. LA RENUNCIA A LA CALIDAD DEMOCRÁTICA DEL AGRUPADO Y OTRAS ANALOGÍAS CON EL SISTEMA ONU. 5. CONCLUSIONES ENLAZADAS CON EL PROYECTO DE INVESTIGACIÓN «CONSTITUCIÓN Y MERCADO EN LA CRISIS DE LA INTEGRACIÓN EUROPEA»: Unión Europea y G-20. 6. ApÉNDICE (Bibliografía, RED E INFORMACIÓN BÁSICA).
\end{abstract}

1 Trabajo realizado en el marco del Proyecto de Investigación Constitución y mercado en la crisis de la integración europea. $\mathrm{I}+\mathrm{D}+\mathrm{i}$ del Programa Estatal de Investigación, Desarrollo e Innovación orientada a los Retos de la Sociedad, del Ministerio de Economía y Competitividad (DER2013-48327-C3-1-R). Investigadores principales: José Luis GARCia Guerrero y María Luz Martínez Alarcón. Agradezco el apoyo ofrecido por el Instituto para la Gobernanza y la Racionalidad del voto, y por la Red Científica Internacional sobre Derecho y Economía. 


\section{EL G-20 PARA EL DERECHO Y PARA LA ECONOMÍA. INTRODUCCIÓN JURÍDICA A UNA «INSTITUCIÓN» INSTRUMENTAL}

Cuando en 2005 los españoles fuimos a votar, favorablemente, en referéndum, algo que denominaron como Proyecto de Constitución para Europa, puede que solo los interesados en el derecho público supieran diferenciar entre lo que consiste realizar un proceso constituyente, y lo que es reforzar con una consulta la decisión de tus representantes/gobernantes para que cierren un tratado internacional de carácter multilateral. Me refiero a este hecho tan palmario de buscada confusión de la ciudadanía hacia una norma de carácter supranacional, como un ejemplo más y ya conocido, de la presencia en nuestras vidas, haciendas y toma de decisiones, de unas macroestructuras internacionales que no terminan de reconocer el déficit de participación de los pueblos que componen los Estados soberanos, demandando mecanismos de acompañamiento. En este caso, como punto de partida de un estudio aproximativo sobre el G-20, de lo que se trata es de exponer ese hecho que consiste en que las estructuras preexistentes de toma de decisiones no solo demandan en ocasiones una búsqueda de legitimidad popular para sus acuerdos: incluso también van a demandar figuras e «instituciones» instrumentales paralelas y flexibles que se destinan, en principio, a mejorar el funcionamiento de entramados organizativos ya constituidos. Ambos ejemplos son la muestra de la tolerada imperfección de los mecanismos representativos internacionales y supranacionales.

Cuando hace una década sucedía esa llamada a referéndum para la «constitución» europea, ya llevaba varios años funcionando una serie de reuniones internacionales conocidas por «G», con la finalidad de servir de foros de toma de decisión política en asuntos de relevancia económica mundial, es decir, de inseparable interés con la mayor parte de decisiones que afectan a los ciudadanos de los Estados allí representados, y por el peso cualitativo y cuantitativo de los mismos, también de una gran parte de habitantes del planeta. El G-20, evolución organizativa de otros grupos muy conocidos que aglutinaban a las potencias mundiales de finales del siglo XX, nace como foro destinado a incorporar la voz gubernativa de los países que por economía y población suman la mayor parte de personas bajo sus fronteras y la más absoluta preponderancia económica sobre las otras decenas de países que no están representados. Su origen viene del propio impulso de las potencias occidentales tradicionales del último tercio del siglo pasado, el G-7, para el tratamiento de asuntos financieros relativos a la búsqueda de la estabilidad económica mundial, y sus efectos en el crecimiento y el desarrollo. La impronta económica de la idea es indiscutible, pues nace articulada formalmente en la reunión de responsables de Economía del G-7 de 26 de septiembre de 
1999, siguiendo las directrices de la cumbre de Colonia de junio de ese mismo año, y culminando con otra cumbre de inauguración los días 15 y 16 de diciembre de 1999 en Berlín. Lo componen los miembros de los «ges» 7 y 8 precedentes: Estados Unidos, Alemania, Reino Unido, Francia, Japón, Canadá, Italia y Rusia, más las adiciones que lo separan de la visión parcial, occidentalista y cuantitativamente no representativa. Así, se suman las demás potencias económicas emergentes y/o de planteamientos económicos dispares con la línea de economía de mercado preponderante en el G-8: China, India, Australia, Corea del Sur, Indonesia, México, Brasil, Turquía, Argentina, Arabia Saudita y Sudáfrica. Para cerrar el círculo de la influencia económica de otras naciones de economía y población más pequeña, el vigésimo miembro es la Unión Europea. El concierto internacional se articula, en congruencia con sus fines, a través de la participación de los responsables gubernamentales de finanzas y de bancos centrales. Oficialmente, se refieren a los países que provienen del G-7 más parte de la Unión Europea, como industrializados, y al resto como «emergentes».

La organización de este concierto intergubernamental es bien sencilla: a. el objetivo de estabilidad económica se concreta a través del impulso de cualesquiera medidas que redunden en ello, tanto promocionales como de persecución de prácticas ilícitas. b. Se reúnen anualmente c. También cada año rota la presidencia según el país organizador de la cumbre. Al modo conocido en la Unión Europea, se pretende una estabilidad de continuación compuesta por una troika conformada por el convocante anual del evento, el que lo presidió el anterior y el que lo hará el siguiente. d. Invitan a otros grupos ${ }^{2}$, organizaciones $^{3}$ y terceros países a las cumbres, en ocasiones de manera permanente, como sucede con España desde $2015^{4}$. Y como máxima muestra de su impronta económica, cumbres, reuniones preparatorias o grupos de trabajo, cuentan con la presencia activa de representantes de los organismos mundiales con competencias/influencias económicas y financieras: Naciones Unidas, Fondo Monetario Internacional, Banco Mundial, OCDE, Organización Internacional del Trabajo, Financial Stability Board, etc.

No es mi intención, desde estas páginas, facilitar al lector información divulgativa, que bien podrá encontrar en un apéndice posterior. Las líneas anteriores intentan mostrar con la elocuencia de la brevedad, el principal beneficio y la más palmaria dificultad que acompaña al G-20: por una parte, en cuanto a lo positivo, ha de resaltarse la existencia de un ámbito de discu-

${ }^{2}$ Por ejemplo, a foros representativos de la sociedad civil (C20) o los negocios (B20).

3 El caso de ASEAN (Association of South-East Asian Nations).

4 Ya representada como miembro de la Unión Europea, también ha sido país invitado con habitualidad o ha ejercido formalmente representando al resto de sus socios durante la presidencia rotatoria de la UE. 
sión flexible donde tratar las cuestiones de relevancia económica mundial (es decir, todo lo que afecta a la humanidad, directa o mediatamente), sin reglas procedimentales más allá de las convenciones descritas y las que marque la cortesía, sin estructuras, personal permanente, votos o vetos. En ellos se habla de mercados, crisis, prioridades, preocupaciones e incluso de las organizaciones constituidas (como el Fondo Monetario Internacional). Ello posibilita que, ante relevantes amenazas a la economía mundial como el escándalo de Lehman Brothers en 2008, exista cierta capacidad de respuesta global. En cuanto lo negativo, obvio es decirlo desde un estudio desde el campo del derecho constitucional, aparece la superación de esquemas organizativos internacionales, que parecen ser reconocidos como inoperantes en las tomas de decisiones; y en definitiva, la falta de aplicación de esquemas de control y participación de la ciudadanía de los países miembros a través de sus parlamentos, profundizando una línea de cooperación internacional donde se prima el ámbito gubernativo y donde ni siquiera queda la presencia de la voluntad de los habitantes de los países miembros (y menos de los que no lo son), en la supervisión de tratados y convenios de eficacia multilateral pero con clara incidencia nacional.

De cualquier manera, estas páginas no pretenden recrearse en una crítica al respecto invocando la necesidad que todo jurista del derecho público tiene de llamar a la organización democrática que procura una toma de decisiones legítima: tan solo como punto de partida hay que constatar que, si el siglo XX ha sido el de la construcción de un modelo internacional de convivencia tanto mundial como regional, el siglo XXI parece presentarse desde su mismo inicio como el de la deconstrucción. El G-20 no es otra cosa que la expresión, puede que bastante realista e irrefutable, del fracaso provocado por la lentitud del entramado de suma de Estados constituido por la Organización de Naciones Unidas, así como del desfase práctico que surge de la necesidad de tomar decisiones en los centros de poder, que sí influyen en la economía global y que no están (o el peso que reflejan es bajo) en el más de un centenar de países no representados en este foro de industrializados y emergentes. El problema jurídico-público, no obstante, es que este conjunto de naciones, cuando en ellas su sistema sea democrático y lo permita, debería articular sus aportaciones y cualquier proceso de toma de decisión con sujeción a los cauces emanados del principio de legalidad que reviste la adopción ad intra de los actos de sus gobiernos, y la aplicación de su orden jurídico de habilitaciones y controles ante los compromisos adquiridos a nivel internacional. Es por tanto en el ámbito del derecho interno donde, en cierto modo, pudiera compensarse la superación de los esquemas preestablecidos de toma de decisiones que parecían imponerse durante el siglo XX a través de una numerosa y compleja red de tratados y convenios; con sus correspondientes organizaciones. 
Ahora bien, tampoco esta primera conclusión puede desatender la compleja realidad de la toma de decisiones en la economía mundial, muy especialmente en los años de crisis económica precedente: el G-20, de una parte concilia sus funciones con la coexistencia del orden internacional que sus impulsores han apadrinado y que los sumados han tolerado. Y de otra, da visibilidad a lo que hasta el momento se trataba a puerta cerrada en contactos de todo tipo y con presencia de grupos de presión de toda índole. Su actuación, pues, podría recordar vagamente a la de la Junta de Portavoces de nuestro derecho parlamentario, cuando prepara, desbroza y simplifica asuntos que luego ha de tratar el pleno de una cámara representativa. Si la concertación que nace de las reuniones anuales supone marcar prioridades en las organizaciones internacionales y en los instrumentos financieros y económicos ya articulados, el G-20 requiere la confianza ciudadana. A fin de cuentas, su esencia es, precisamente, no querer ser una «institución», para ganar así en margen de libertad y confrontación de ideas, algo loable siempre que desde el derecho se subraye la necesidad de articular esos posibles beneficios por los cauces establecidos. La máxima expresión de los poderes políticos y económicos puede llegar a ser compatible con una articulación jurídica más acabada, y en ocasiones ya conseguida, en instituciones preexistentes.

En resumen, desde el punto de vista formal, creo que el Grupo de los 20 es un mecanismo instrumental, de escasa articulación institucional pero de amplísima capacidad de influencia, que con su definición de líneas de política económica y financiera agiliza la toma de decisiones por parte de países y organizaciones internacionales. Y desde el material o de contenido, comparto con CARRERA, que lo más relevante es cómo procura la apertura de la concertación a las economías emergentes que se esconden tras estados densamente poblados 5 .

${ }^{5}$ CARrera, J., «El G20, la crisis y el rediseño de la arquitectura financiera internacional», Banco Central de la República Argentina, Investigaciones Económicas, BCRA n. ${ }^{\circ}$ 2009/45, Noviembre de 2009. Pág. 2: «Una de las notas más originales de la crisis actual es el ámbito o foro de discusión multilateral decidido para su tratamiento: el G20, del cual participan tanto economías avanzadas como emergentes. A diferencia de lo ocurrido en los anteriores rediseños de la AFI, los países emergentes tienen esta vez la oportunidad de participar en el debate y en la toma de decisiones. La presencia de estos países en el G20 es un reconocimiento de su creciente importancia relativa (representan más del $30 \%$ del PIB mundial y han explicado, aproximadamente, un $60 \%$ del crecimiento global en los últimos años) y de su mayor protagonismo en la arena comercial, monetaria y financiera internacional. Este grupo nació en 1999 a raíz de la crisis asiática. Su objetivo inicial era promover un ámbito en el cual las principales economías avanzadas y emergentes dialoguen a nivel político y técnico sobre el mejoramiento del sistema financiero y también sobre temas relacionados con el crecimiento de largo plazo como, por ejemplo,las políticas para morigerar el calentamiento global». 


\section{2. ¿LA ORGANIZACIÓN DEL G-20 POSIBILITA SUS FINES INSTITUCIONALES? CONGRUENCIA CON SUS FUNCIONES}

En anteriores estudios me he referido a la necesidad de depurar del ordenamiento jurídico de un Estado todas las instituciones que no tengan encomendada una función que, de un modo u otro, influya en los derechos y libertades de las personas ${ }^{6}$. Creo que es una medida acertada la aplicación de este parámetro de organización institucional en todo momento, y no solo en tiempos de crisis, que es cuando se buscan las simplificaciones por motivos de contención del gasto público. Que cada institución u órgano cumpla unos cometidos, y solo esos, que redunden, por aplicación o protección, en el ámbito de nuestros derechos, sirve para recordar la instrumentalidad de las instituciones y la preeminencia del corazón democrático constituido por el sistema de libertades, sobre la estructura «ósea», representada por los órganos de un país o de una comunidad internacional.

Las instituciones económicas previstas en las constituciones de los países democráticos se ajustan a este perfil, y su finalidad planificadora, preventiva, recaudatoria, solidaria o redistributiva; tan solo tiene como finalidad el abastecimiento de los fondos necesarios para realizar los derechos, ya sea desde los poderes públicos o procurando el ambiente social para su materialización (empleo, seguridad mercantil, etc.). En una estructura internacional rige el mismo principio de fundamentación: Naciones Unidas o Unión Europea, mantienen una serie de finalidades que convergen en procurar derechos a las personas de sus Estados miembros, careciendo de interés un entramado institucional de este tipo, y dentro de él, cualquiera de sus órganos, si su fin último no es redundar en la mejora de las condiciones de vida que en conjunto facilita procurar los derechos humanos reconocidos. El desarrollo en las últimas décadas de organismos especializados en materia económica y financiera como el FMI o el Banco Mundial, no se sale de este patrón y tan solo busca la operatividad, según parece irrealizable de ordinario, en foros gubernamentales multilaterales de competencia genérica. La toma ágil de decisiones económicas y crediticias, y no solo la especialización, están en el origen de este tipo de órganos. Ahora bien, lo que cabe preguntarse (aunque no pueda responderse en las limitaciones de espacio que se imponen en este estudio) es si estas instancias económicas mundiales tienen una relación de dependencia y control con estructuras de participación estatal similares a las que órganos especializados en materia económica tienen con parlamentos y gobiernos dentro de los Estados; o si por el contrario, la autonomía funcional

${ }^{6}$ Belda PÉRez-Pedrero, E., Los derechos de las personas y las funciones del Estado como límite a la supresión de instituciones. La crisis económica y la reforma del Estado. Centro de Estudios Políticos y Constitucionales, Madrid, 2014. 
y de toma de decisiones es de tal calibre que los propios mecanismos de control terminan por atenuarse y el engarce con la voluntad de los Estados soberanos que las promueven se debilita. El problema es si cabe mayor, cuando la influencia en la toma de decisiones se experimenta no solo desde las estructuras estatales competentes sino también desde grupos de presión económica, multinacionales, o desde cualquier ente no gubernamental.

La articulación del G-20, en este sentido de la finalidad funcional, no tiene mancha: primero porque no se constituye como institución internacional pues es solo una simple concertación donde no han de barajarse criterios de sostenibilidad económica o eficiencia en la gestión de fondos. Segundo, porque sus cometidos inciden de forma absolutamente decisiva en la materialización de los derechos, al trabajar sobre su provisión económica tanto desde el punto de vista de la financiación como desde el de la cobertura sostenible y previsión de su mantenimiento. Por último, los gobiernos actúan directamente, con lo cual sus responsables rinden cuentas en el ámbito que dispone la legislación interna de sus Estados para el control de las acciones derivadas de la competencia en política exterior.

Si la actuación del G-20 es satisfactoria a los fines fundacionales para los que fue concebido, lo habrán de responder los analistas económicos internacionales o las ciencias económicas, e incluso las ciencias políticas 7 . Desde los primeros parece haber una evaluación anual dependiente de la actividad derivada de sus cumbres, mientras que las segundas analizan en su caso, global o parcialmente, los frutos de sus lineamientos económicos una vez que se materializan tras las decisiones de instituciones internacionales o nacionales que los adoptan. Unos y otros resaltan, no siempre de forma positiva, la informalidad de las reuniones del grupo: lo que de beneficio conlleva la falta de formalidad, es claro que se corresponde con la ausencia de instrumentos para revertir con eficacia cualquier disfuncionalidad del sistema ${ }^{8}$. El trasfondo económico de la toma de decisiones se mueve por dos exigencias: una general y siempre presente, que es la interdependencia de las economías mundiales, que obliga a la adopción de medidas comunes, y otra de aparición cíclica o regional, ocasionada por las crisis inherentes en el sistema económico.

Lo que al derecho público le llama la atención de este planteamiento en el ámbito de las competencias económicas (igual que desde el punto de vista

7 Entre otros: Colomer, J. M., El gobierno mundial de los expertos, Anagrama, Barcelona, 2014. JoKeLA, J., «The G-20: A pathway to Effective Multilateralism?», en Chaillot Papers n. ${ }^{\circ}$ 125, ISS Unión Europea, abril de 2011. NAKAGAWA, Junji, International Harmonization of Economic Regulation, Oxford University Press, 2011. Postel-Vinay, K., The G-20: A New Geopolitical Order, Palgrave Macmillan, Londres, 2014.

${ }^{8}$ En este sentido SteinberG, F., «Por qué hay que esperar poco del G-20» Expansión. 12 de noviembre de 2014. 
institucional, en el apartado anterior lo acabo de mencionar, era la debilidad de los lazos de control ciudadano), es sin duda el aspecto de cesión de soberanía que ya ha sido ampliamente tratado al abordar la traslación de toma de decisiones económicas hacia organismos supranacionales regionales, y que el tiempo sigue demostrando como problema no resuelto9. Así, cualquier planteamiento de organización internacional que pretenda cierto grado de eficacia en la planificación y ejecución de políticas económicas o en la prevención de efectos adversos de las mismas, demanda para su éxito de un sustancial trasvase de ámbitos competenciales económicos en sentido ciertamente extenso (medidas presupuestarias, financieras, crediticias, de gasto, incluso de ejecución de todo tipo de políticas sociales), y con ello renunciar a una de las partes más preciadas del haz de competencias estatales derivados de la soberanía de los Estados. Si ese paso ha sido, o está siendo (véase el caso de Grecia en 2015) dificultoso para los países que hace décadas teníamos esa posibilidad en nuestras previsiones internacionales, incluso incorporadas al propio texto constitucional (art. $93 \mathrm{CE}$ ), piénsese en aquellos otros, miembros del G-20, que por su condición geográfica o política levantan sobre sus espacios de soberanía un velo protector más denso (China, India). En el G-20 se renuncia de plano a este planteamiento de cesiones, e incluso la propia dinámica de adopción de acuerdos impide que se cuestione lo más mínimo el ámbito de competencias económicas de los Estados miembros, al carecer de una reglamentación interna de votos donde las mayorías signifiquen algo, o coarten tanto las decisiones finales de sus participantes en otros foros internacionales constituidos, como las decisiones en la legislación propia y la ejecución interna de los lineamientos económicos alcanzados.

\section{LA TEMÁTICA ECONÓMICA COMO EJE DE LA EXISTENCIA DEL G-20. LA PRESENCIA DEL DERECHO EN LA ECONOMÍA. ¿Y DE LA ECONOMÍA EN EL DERECHO?}

La significación y protagonismo económico de esta pseudoestructura internacional que han bautizado como G-20 es inobjetable ${ }^{10}$, siendo uno de

9 Ver el enfoque de Balaguer CAllejón, F., «Crisis económica y crisis institucional de Europa», En Revista española de Derecho Constitucional n. ${ }^{\circ} 98$, págs. 91 a 107, mayoagosto de 2013. Desde el punto de vista de problemas concretos, un ejemplo en GIL IBANEZZ, A., «Principio de primacía del derecho europeo y Constitución española: límites y consecuencias de la cesión de soberanía por la nueva Constitución europea», en Gaceta Jurídica de la Unión Europea y de la Competencia n. ${ }^{\circ}$ 232, págs. 3 a 16, 2004.

${ }^{10}$ CARrera, J., «El G20, la crisis y el rediseño de la arquitectura financiera internacional», ob. cit., pág. 1: «Todas las grandes crisis han requerido reformular la Arquitectura Financiera Internacional (AFI). El G20, constituido como foro de discusión y acción frente a la crisis, concentró su trabajo en dos áreas: primero, la coordinación de las políti- 
los exponentes primeros de la respuesta actual ante las crisis cíclicas. La cuestión es, como se viene adelantado, determinar qué posición debe adquirir el orden jurídico para articular estas formas de actuar de los agentes económicos, $\mathrm{y}$ de los propios agentes políticos cuando han de actuar en el campo de la economía. A la inversa, parece clara, y la necesaria presencia de la economía en la toma de decisiones articuladas por una norma, ha sido resaltada desde diversos puntos de vista ${ }^{11}$ como elemento imprescindible para la consecución de decisiones razonables y sostenibles en cualquier sociedad avanzada.

La pregunta que se puede plantear, por ello, al hilo de la filosofía organizativa del G-20 es si debe estar el derecho, entendido como conjunto de reglas del juego, presente en la economía. La respuesta es afirmativa pero debe razonarse desde distintos frentes. Por una parte, el G-20 como tal, toma una serie de decisiones o acuerdos políticos, o de política económica, en un nivel ajeno a los procesos de decisión normativa o de directrices nacionales o internacionales. La buscada posición de comodidad en sus esquemas, que le sitúa al margen de la dinámica de necesaria sujeción de todos los actos a un soporte jurídico, es el marco buscado por sus componentes para la agilidad,

cas macroeconómicas para salir de la crisis y segundo, la reforma de la AFI tanto en lo concerniente a las regulaciones financieras como a las organizaciones multilaterales de crédito y supervisión. Quedó fuera de la agenda atender otros aspectos críticos de la AFI como las reglas de funcionamiento del sistema monetario internacional. En relación a esta cuestión, es materia de debate la situación actual de «no régimen» que generó fenómenos como los desbalances globales, careció de reglas claras respecto al rol del dólar en la provisión de liquidez internacional y como reserva de valor, y que tampoco tuvo un prestamista de última instancia. El hecho de que se abriera la discusión del rediseño de AFI a nuevos actores como los países emergentes ha sido muy positivo. El desafío es lograr que este marco persista cuando la crisis sea superada para que estos países puedan tener una inserción internacional menos asimétrica».

${ }^{11}$ Posner, R., Economic Analysis of Law, Little Brown, New York, 1973. En España, las disciplinas que se encargan en Ciencias jurídicas y Económicas de esta materia son la Economía Política y la Hacienda Pública, de modo principal. Como aproximación al tema: CABriLlo, F., «¿Por qué un análisis económico del derecho?». En Revista de Occidente n. ${ }^{\circ}$ 58, págs. 37 a 46, 1986. DuRÁn Lalaguna, P., Una aproximación al análisis económico del derecho, Comares, Granada, 1992. Mercado Pacheco, P., El análisis económico del derecho. Una reconstrucción teórica, Centro de Estudios Constitucionales, Madrid, 1994. Polinsky, M. A., Introducción al Análisis económico del Derecho, Ariel, Barcelona, 1985. Pastor, S., «Una introducción al Análisis Económico del Derecho», en Hacienda Pública Española, n. ${ }^{\circ}$ 89, págs. 137 a 157 (1984). Del mismo autor: Sistema jurídico y economía. Una introducción al Análisis Económico del Derecho. Tecnos, Madrid, 1989. Torres LóPez, J.; El Análisis Económico del Derecho. Tecnos, Madrid, 1987. En Filosofía del Derecho, entre otros: CAlSAMIGLIA, A., «Eficiencia y Derecho», en Doxa n. ${ }^{\circ}$ 4, págs. 267 a 302, 1987. Hierro, L., «La pobreza como injusticia (Dworkin v. Calabresi)» en Doxa, n.os 15 a 16, págs. 945 a 969, 1994. 
al menos pretendida, en la toma de decisiones. Donde se produce el sometimiento al principio de legalidad y sometimiento al sistema de fuentes del derecho, es en el ámbito interno de cada uno de esos países participantes: así, los compromisos internacionales de los mandatarios y responsables económicos que asisten a las reuniones, solo se materializan cuando en sus respectivos ámbitos de competencia concretan los acuerdos conseguidos en estos grupos, sea este G-20 o en los otros G's coexistentes, a través del acto normativo o ejecutivo procedente, y de acuerdo a los principios y reglas de su derecho interno, o en el caso de la Unión Europea, en el de su derecho comunitario.

Por otra parte, la discrecionalidad decisiva que podría mostrarse en las cumbres del G-20 en abuso del marco abierto con el que se plantea el foro, no es tal. Como se acaba de reseñar, los mandatarios actúan con arreglo a las normas desde las que extraen su mandato en materia de política exterior o económica, y además, la presencia de las instituciones internacionales de toda índole a la que nos hemos referido, llevan al foro intergubernamental al mismo concierto mundial, conocido y establecido, que impide la disociación entre las finalidades de este grupo y las perseguidas por esas instancias internacionales constituidas. Así, el G-20 se abstiene de suplantar, por superación cualitativa y económica, a las organizaciones mundiales o regionales. Ese peligro podría parecer prima facie inquietante: ante un anquilosamiento del orden surgido tras la II Guerra Mundial, y rechazada la dinámica de poder efectivo de una o dos potencias mundiales, se constituye un nuevo orden de facto en el que los más grandes relevan en la práctica al concierto de naciones ya constituido. No parece que el G-20 esté generando esa realidad, pues si las principales potencias marcan calendarios y finalidades, ocurre y ocurría al margen de la existencia de los G's. Además, la propia amplitud del G-20 frente a los precedentes G's 7 u 8 , introduce un factor de diversidad y concierto que le aproxima más a instituciones articuladas procedentes (las del marco ONU) que a la dinámica de políticas internacionales basadas en bloques, que fue propia de la segunda mitad del siglo XX.

Por todo ello, el G-20 se presenta como un cuerpo distinto, ante el orden internacional, pero no es ni ajeno ni extraño al mismo. Sus decisiones no chocan ni violentan las del orden previamente constituido pues se articulan en una fase previa de concertación que puede o no tener efectos. Además, como suma de mandatarios que ejercen una serie de funciones previamente establecidas en ordenamientos jurídicos con reglas específicas de competencias y bajo el imperio del principio de legalidad, cualquier reunión del G-20 se aleja de patrones poco deseables de funcionamiento descontrolado. Como subrayaba anteriormente, las posibles dudas de falta de control en origen, de riesgos de influencia por parte de grupos de presión, etc., tienen menos relevancia que cuando su existencia se denuncia sobre las instituciones interna- 
cionales o nacionales que efectivamente deben de tomar las decisiones, y en cualquier caso, la magnitud de la convocatoria desanima algo más a los presuntos interferentes de lo que lo pueda hacer una reunión más reducida de las principales potencias o de las instituciones económicas observadoras.

Hasta aquí se ha dejado constancia de la presencia del orden institucional nacional o internacional, en la estructura organizativa del G-20, pero habría también de sumarse esa aparición en el contenido de las decisiones, en este caso económicas. La respuesta solo cabe desde la continuidad de los planteamientos anteriores: las conclusiones de los participantes en las cumbres anuales, reuniones preparatorias o grupos de trabajo se articula desde el respaldo constitucional, legal o estatutario que les ofrecen las fuentes del derecho a la que su representación se sujeta. Los lineamientos ofrecidos por los países participantes en estos foros existen para los medios de comunicación y mercados financieros (que no es poco), pero no para la realidad hasta tanto no se concretan. Es claro, además, que las recomendaciones o conclusiones pueden seguirse por todos los participantes, solo por parte, por ninguno, o por terceros países; siendo el momento de la traslación a los Estados y organizaciones, el único relevante a efectos jurídicos. Como acabo de plantear, es obvio que en la práctica la incidencia en los mercados puede ser notoria, no obstante, pero el derecho no puede (y a lo mejor no debe) remediarlo.

\section{LA RENUNCIA A LA CALIDAD DEMOCRÁTICA DEL AGRUPADO Y OTRAS ANALOGÍAS CON EL SISTEMA ONU}

Los españoles hemos conocido históricamente la inhabilitación que supone padecer un régimen autocrático, y que nuestro Estado quiera participar en una organización internacional, siendo negada nuestra incorporación por la ausencia de un orden interno basado en leyes democráticas. La pluralidad de naciones que impulsó la Organización de Naciones Unidas de 1948, posibilitaba en aras de un entendimiento global y de otras razones prácticas, la presencia de todos los Estados constituidos. No así otras organizaciones internacionales de ámbito regional como el Consejo de Europa, o supranacional, como la Unión Europea. El G-20, como demuestra su trayectoria, se centra en los temas económicos soslayando, más bien admitiendo como un hecho, la diversidad organizativa de sus participantes y el distinto estatuto jurídico de los habitantes de los países representados. Sin necesidad de señalar a qué países nos referimos, lo cierto es que el G-20 renuncia a exigir lo que se suponía como requisito superado en su primer origen, como G-7 (Se trata de los países más avanzados en todos los campos y no solo en el económico, donde el desarrollo ha seguido al crecimiento, democráticos, en congruencia internacional en el ideario de respeto de los derechos humanos que reposa tras los Estados sociales y democráticos de derecho). Nos enfrenta- 
mos entonces, al lamentable realismo que constituye la ausencia de regímenes democráticos sobre el gobierno de centenares de millones de personas.

No parece lógico que esa exigencia, ausente en instituciones internacionales previas, haya de planear sobre un ámbito de intercambio de opiniones. La finalidad misma de previsión de los problemas mundiales surgidos de las crisis económicas, es una pura contradicción con las concepciones en la materia de algunos de los participantes en el grupo, que al menos en teoría negaron históricamente los principios del mercado que condicionan toda la actividad de este foro. De cualquier forma, si estas líneas están situando el foco de estudio sobre la necesidad del ciudadano de saberse parte de las instituciones y de la toma de decisiones de sus poderes públicos, a través de una suerte de vigencia de los principios de participación y control en todo aquello que les afecta; no quedaría otro remedio que la renuncia a una respuesta conjunta en la evaluación de las decisiones de este G-20. Así, la legitimidad democrática de la toma de decisiones solo puede ser abordada parcialmente y respecto de aquellos Estados o uniones de Estados donde el derecho constitucional confiere de forma certera y reconocible a sus mandatarios la capacidad de tomar decisiones en las áreas afectadas por la economía y la política exterior. Cualquier test de «democraticidad», o de representación y control, que quisiera aplicarse a este foro de naciones en su conjunto, no tendría resultado positivo, como tampoco razón de ser.

Qué duda cabe, no obstante, de la influencia en millones de personas de los resultados obtenidos en las cumbres, y de la inconsistencia filosófica que surge de la confrontación del modelo en el que unos participantes guardan tras de sí un origen democrático en sus actos, y otros imponen a los habitantes de sus territorios las medidas adoptadas. En este sentido, se retrata el G-20 como una muestra más de su capacidad de ser espejo del sistema actual de naciones, donde la falta de armonización en idearios organizativos y en carencias de mínimos denominadores en el respeto práctico de los derechos, es la nota dominante. Un buen ejemplo, al que aludíamos al describir el régimen organizativo y que procede traer a colación, es el del reparto de presidencias/cumbres que se instauró en el grupo desde la cumbre de Seúl de 2010, y que recuerda la dinámica de bloques o de intereses que se percibe en las experiencias organizativas internacionales que protagonizaron la descolonización. La decisión de reglamentar la organización de las presidencias, dividiendo en grupos a los países miembros no parece que sea la de repartir el peso entre bloques, pues a todos alcanza la rotación. Más bien sería la de dar estabilidad a la convocatoria de reuniones, sin dejar al arbitrio de la oportunidad geopolítica de los mandatarios, la concertación anual de este evento. Así, los grupos se numeran del 1 al 4, siendo el primero el constituido por Arabia Saudita, Australia, Canadá y Estados Unidos. El segundo es el de India, Rusia, Sudáfrica y Turquía. El tercero el de Argentina, Brasil y Méxi- 
co. El cuarto lo forman Alemania, Francia, Italia y Reino Unido. Y el quinto, el compuesto por China, Corea del Sur, Indonesia y Japón. El criterio geográfico es evidente en los tres últimos grupos, el latinoamericano, el europeooccidental y el asiático, pero no en los dos primeros. Las últimas presidencias a partir de Seúl 2010 fueron cambiando de grupo (Francia, México, Rusia y Australia; de 2011 a 2014). La presidencia China opera en este año 2016 (Hangzhou), que sería un nuevo grupo, pero en 2015 se realiza en Turquía, que está en el grupo de Rusia, que presidió en 2013, por lo cual la correlación no opera automáticamente, ya que ha habido grupos que repiten desde ese acuerdo de 2010, antes de la participación de países adscritos a otros, sin que transcurran cinco años.

\section{CONCLUSIONES ENLAZADAS CON EL PROYECTO DE INVESTIGACIÓN «CONSTITUCIÓN Y MERCADO EN LA CRISIS DE LA INTEGRACIÓN EUROPEA»: UNIÓN EUROPEA Y G-20}

En este último epígrafe ha de hacerse una referencia a dos asuntos básicos, que son los que justifican este estudio como contribución al amplio proyecto de investigación de referencia, una vez que se han realizado las anteriores consideraciones.

La primera cuestión es instrumental o formal: La Unión Europea es parte del G-20 como un solo miembro, participando a través de las autoridades de países que componen dicha Unión, y representando a su vez a una multiplicidad de Estados (que para mayor complicación están en algún caso ya en el G-20). La segunda es de fondo: ¿afecta el G-20 a la crisis o replanteamiento de la Unión Europea, que es el objeto del presente proyecto de investigación, a nivel de toma de decisiones, tanto institucionales como de política económica? La respuesta habría de darse desde el ámbito de otros campos como la Sociología o la Economía, pero aquí ha de constatarse en una aproximación que responda a una visión completa de las relaciones entre G-20 y Unión Europea, de la similitud de objetivos, aunque parcial, entre ambas figuras, a pesar de la no institucionalización propia del G-20.

a) En la fundación del G-20 aparece la impronta de las potencias occidentales como ocurría en el precedente G-7. Los países más desarrollados han sido los que han constituido en el último tercio del siglo XX el eje representativo de las potencias modelo de la economía de mercado, Estados Unidos, Japón, Canadá, Reino Unido, Alemania, Francia, Italia. Los cuatro últimos son países miembros de la Unión Europea, y de entre ellos, los tres últimos fueron los impulsores en los años cincuenta del pasado siglo de la estructura de las Comunidades Europeas. La sustancialidad e individualidad como agente de política inter- 
nacional que logra la Unión Europea a partir de 1992, coloca a esta experiencia supranacional al primer nivel de las potencias económicas mundiales más allá del cómputo de riqueza u operaciones de las cuatro potencias señaladas, pues varios millones de personas más y una veintena de países medianos y pequeños, aportan un valor adicional en este campo. Ese plus o suplemento que la posiciona en la economía global como agente de primer orden, es la toma de decisiones conjunta en el ámbito de la economía, así como la puesta en común, al menos en las finalidades que interesan al G-20, de instituciones económicas que actúan en nombre de todos los miembros.

El peso de los países de la Unión Europea en el G-20, a pesar de esta posible duplicidad que supone que varios de ellos puedan estar adicionalmente representados en ese grupo por las instituciones supranacionales europeas en su conjunto, es notablemente inferior al que gozan en los G's 7 y 8 fundados con anterioridad. Es la muestra elocuente del cambio de siglo y la asunción por parte del continente europeo de un plano secundario ante los países emergentes con elevadas cifras de producción: puede que ya camine a la posición de acólito, que es el que, parece, le terminará correspondiendo al cabo de unas décadas. La presencia de toda la Unión Europea en el G-20 se articula por sus reglas internas, participando los representantes comunes de política exterior y economía, así como los del Estado que ostente la presidencia rotatoria y en su caso, los otros dos que componen la troika que da estabilidad a las rotaciones. Países medianos de la Unión como España, son sumados por diversos medios al G-20, por cesión de asiento de los socios o ya recientemente como país invitado permanente ${ }^{12}$.

12 «La primera participación española en el G-20 tuvo lugar en la cumbre extraordinaria de noviembre de 2008 en Washington cuando el entonces presidente de Francia, Nicolás Sarkozy, cedió a España uno de los dos puestos que le correspondían como presidente de turno de la Unión Europea y como miembro del G-8. Tras la cita de Washington, España fue invitada oficialmente para asistir a la cumbre extraordinaria de Londres de abril de 2009, con el primer ministro británico Gordon Brown como anfitrión. Después de participar en la cumbre de Londres, España consideró consolidada su posición en el G-20 como corresponde a su peso político y económico en la comunidad internacional. España también estuvo en Pittsburgh, en septiembre de 2009, para participar en la cumbre extraordinaria presidida por Barack Obama, anfitrión de la cita. En esa ocasión España ejercía la presidencia de turno de la Unión Europea. Posteriormente, España ha asistido a la cumbre extraordinaria de Toronto, en junio de 2010. Durante la presidencia de Corea del Sur, tuvo lugar la cumbre extraordinaria de Seúl, en noviembre de 2010; en ella, el país anfitrión apoyó la posibilidad de que España formara parte de un G-20 ampliado. España ha participado en las últimas cumbres, celebradas en Cannes (Francia), en noviembre de 2011, en Los Cabos (México) en junio de 2012, y en San Petersburgo (Rusia) en septiembre de2013». http://www.exteriores.gob.es/ Portal/es/PoliticaExteriorCooperacion/G20YOCDE/Paginas/Presencia.aspx (fecha de con- 
El problema de este esquema en el que podrían surgir, y de hecho surgen habitualmente, intereses económicos contrapuestos y prioridades distintas en orden al gasto, la carga impositiva o múltiples cuestiones financieras; es una cuestión ajena al G-20, al que solo llegará la voz única de la Unión Europea. Ello no es obstáculo para que tales divergencias se reflejen, en el caso de los miembros que la componen, pero que por su propia importancia también han accedido al grupo.

La representación de la Unión Europea en el G-20 se canaliza por las reglas internas de representación exterior, asistiendo a las cumbres el Presidente del Consejo Europeo y el Presidente de la Comisión Europea. A las citas interministeriales acude el ministro competente en razón de la materia, del Estado que ostenta la presidencia rotatoria, el comisario correspondiente en función de la competencia sobre el tema objeto de debate, y una representación del Banco Central Europeo.

b) La segunda cuestión a abordar, y que concluye con la presente aproximación al G-20 por parte del grupo de investigación al que hacíamos referencia en primera página, es la influencia de este concierto económico en el período crítico, institucional y financiero, que se observa en la Unión Europea. Al margen de la evaluación que ha de corresponder, como se ha dicho, a otras disciplinas, desde el derecho constitucional hay que contestar con un «sí»: lo que el G-20 supone, está presente en el debate sobre crisis y reorientación europea. Primero, porque el fortalecimiento en la última década de un «gobierno común» representado por la Comisión Europea y de mandatarios de instituciones como la presidencia del Banco Central Europeo, es en buena medida la consecuencia del proceso globalizador que representa el G-20, y Europa responde al mismo con la consciencia, también en lo organizativo, que solo como bloque puede afrontar la pujanza económica de China o países emergentes como Brasil, Indonesia, India, o nuevamente en la historia continental, de Rusia. Segundo, porque en el origen de la Unión Europea la principal motivación, por más que su exclusividad se desdibujara en atención a potenciar otros criterios de identidad, fue la económica:

sulta, 25 de mayo de 2015). En la actualidad España es invitado permanente, siendo el único Estado con ese status: $<<$ Each year, the G20's guests include Spain (a permanent invitee); the Chair of ASEAN; Global Governance Group; two African countries (the chair of the African Union and a representative of the New Partnership for Africa's Development (NE$P A D)$ ) and a country or countries invited by the presidency, usually from its own region. Since the first leader-level meeting of the G20 in 2008, guests have included Benin, Brunei, Cambodia, Chile, Colombia, Equatorial Guinea, Ethiopia, Kazakhstan, Malawi, the Netherlands, Spain, Switzerland, Thailand, the United Arab Emirates, Vietnam, Mauritania, Senegal, Myanmar and New Zealand» https://g20.org/about-g20/g20-members/g20world/ (fecha de consulta, 25 de mayo de 2015). 
crecimiento, estabilidad, desarrollo, racionalización de criterios monetarios y crediticios, y prevención o amortiguación de crisis cíclicas. Su modelo ha sido muchas veces una referencia para el resto de la comunidad internacional y aunque los intereses que puedan salir del G-20 (si es que estando en él un número tan amplio y diverso de Estados, cabe pensar que se llegue a un interés coincidente ${ }^{13}$ ) no sean concurrentes con los circunstanciales de la Unión, los Estados asistentes al grupo van buscando (para sí mismos y se supone que para todo el orbe) ese mismo eje constituido por crecimiento/desarrollo/prevención de crisis.

\section{APÉNDICE (BIBLIOGRAFÍA, RED E INFORMACIÓN BÁSICA)}

\section{a) Bibliografia y otras fuentes escritas}

AleXANDER, N., «Introducción al G20», Heinrich Boell Foundation-US, ed. de junio de 2011.

ANTUÑANo MARURI, I., «Las decisiones del G-20 y su impacto sobre los países más débiles» En Documentación Social n. ${ }^{\circ}$ 158, julio-septiembre de 2010.

Balaguer CALLejón, F., «Crisis económica y crisis institucional de Europa» En Revista española de Derecho Constitucional n. ${ }^{\circ}$ 98, págs. 91 a 107, mayo-agosto de 2013.

BANCO Mundial, Postcrisis growth and development. A development agenda for G-20. Banco Internacional para la Reconstrucción y el Desarrollo/Banco Mundial, Washington D.C., 2011.

Belda Pérez-PEDRero, E., Los derechos de las personas y las funciones del Estado como límite a la supresión de instituciones. La crisis económica y la reforma del Estado. Centro de Estudios Políticos y Constitucionales, Madrid, 2014.

BergGruen, N.; Gardels, N., Gobernanza inteligente para el Siglo XXI. Una vía intermedia entre occidente y oriente. Taurus, Santiago de Chile, 2013.

Bernanke, B., «The Macroeconomics of the Great Depression: A Comparative Approach» En Journal of Money Credit and Banking, vol. 27 (1), 1-28, 1995.

BuCHANAN, J.; BrenNan, G., La razón de las normas. Economía política constitucional. Unión Editorial, Madrid, 1987.

CARRERA, J., «El G20, la crisis y el rediseño de la arquitectura financiera internacional», Banco Central de la República Argentina, Investigaciones Económicas, BCRA n. ${ }^{\circ}$ 2009/45, Noviembre de 2009.

13 «Los países emergentes tienen hoy visiones sobre la regulación financiera, los mecanismos de ajuste en el sistema monetario internacional o las mejores prácticas en política comercial que son muy distintas a las de los países avanzados. Incluso tienen una visión sobre cómo debería funcionar (y regularse) el capitalismo que dista mucho de la dominante en Washington o Londres. Además, tras décadas de liderazgo intelectual y político anglosajón en asuntos económicos internacionales, la crisis financiera global ha supuesto un importante golpe a la visión norteamericana del capitalismo, lo que ha puesto a Estados Unidos a la defensiva, generando un vacío de poder significativo en el sistema internacional, que ni los países emergentes ni la Unión Europea están dispuestos a ocupar». STEINBERG, F., ... art. cit. 
Casas Pardo, J.; Bru Parra, S. (eds.), Economía y Política, Universidad de Valencia, 1988.

Colomer, J. M., El gobierno mundial de los expertos, Anagrama, Barcelona, 2014.

Garton, T., «Hacia un mundo libre: del G-8 al G-20», En El País, 28 de mayo de 2011.

HeAP, P. C., Globalización y reforma de cumbres. Un experimento de gobernanza internacional, Centro Internacional de Investigaciones para el Desarrollo-Ediciones Mayol, Ottawa (Canadá)-Bogotá (Colombia), 2009.

Jokela, J., «The G-20: A pathway to Effective Multilateralism?», En Chaillot Papers n. ${ }^{\circ}$ 125, ISS Unión Europea, abril de 2011.

NaKagawa, J., International Harmonization of Economic Regulation, Oxford University Press, 2011.

Nelson, R. M., «The G-20 and International Economic Cooperation: Background and Implications for Congress», En Congressional Research Service, US House of Representatives, 9 de diciembre de 2009.

Portes, R., «Global Imbalances», En M. Dewatripont, X. Freixas y R. Portes (eds.): Macroeconomic Stability and Financial Regulation: Key Issues for the G20, Centre for Economic Policy Research, 2009.

Posner, R., Economic Analysis of Law, Little Brown, New York, 1973.

Postel-Vinay, K., The G-20: A New Geopolitical Order, Palgrave Macmillan, Londres, 2014.

SteInBERG, F., «Por qué hay que esperar poco del G-20», En Expansión, 12 de noviembre de 2014.

b) Enlaces $w w w$

1. G-20

https://g20.org/ (fecha de consulta, 2 de mayo de 2016).

2. España en $\mathrm{G}-20$

http://www.exteriores.gob.es/Portal/es/PoliticaExteriorCooperacion/ G20YOCDE/Paginas/Presencia.aspx (fecha de consulta, 2 de mayo de 2016).

3. Derecho y economía

http://www.voltairenet.org/mot120950.html?lang=es (Red Voltaire).

https://www.facebook.com/\#!/economiayderecho (facebook: Red Científica Internacional sobre Economía y Derecho Público).

\section{c) Información básica}

Fuente: https://g20.org/ (fecha de consulta, 2 de mayo de 2016).

1. Miembros:

Alemania

Canadá 


\section{Estados Unidos \\ Francia \\ Italia \\ Japón \\ Reino Unido \\ Rusia \\ Arabia Saudí \\ Argentina \\ Australia \\ Brasil \\ China \\ Corea del Sur \\ India \\ Indonesia \\ México \\ Sudáfrica \\ Turquía \\ Unión Europea}

2. Organizaciones internacionales concurrentes:

The success of the G20 is achieved with support from international organisations, which provide advice on G20 priorities and help G20 members and guests identify policy gaps where actions will have the most impact. Representatives of international organisations are invited to relevant G20 meetings, including meetings of Sherpas, Finance Deputies and working groups. These organisations include:

- the Financial Stability Board (FSB). The FSB, which was established by G20 leaders following the onset of the global financial crisis, coordinates the work of national financial authorities and international standard-setting bodies to develop and promote effective regulatory, supervisory and other financial sector policies.

- the International Labour Organization (ILO). The ILO promotes rights at the workplace, encourages decent employment opportunities, enhances social protection and strengthens dialogue on work-related issues.

- the International Monetary Fund (IMF). The IMF works to foster global monetary cooperation, secure financial stability, facilitate international trade, promote high employment and sustainable economic growth, and reduce poverty around the world.

- the Organisation for Economic Co-operation and Development (OECD). The OECD promotes policies that improve the economic and social well-being of people around the world. It works with governments to 
understand what drives economic, social and environmental change, with a focus on analysing and comparing data to predict future trends.

- the United Nations (UN). The UN is committed to maintaining international peace and security, developing friendly relations among nations and promoting social progress, better living standards and human rights.

- the World Bank. The World Bank is a vital source of financial and technical assistance to developing countries around the world. It is not a bank in the ordinary sense but a unique partnership to reduce poverty and support development.

- the World Trade Organization (WTO). The WTO provides a forum for negotiating, implementing and monitoring agreements aimed at reducing obstacles to international trade and ensuring a level playing field, contributing to economic growth and development.

\section{Invitados:}

Each year the G20 president invites guest countries to attend the Leaders' Summit to participate in member discussions about the agenda.

Inviting guest countries gives non-members an opportunity to bring their views to the G20 table. The selection of guests reflects the G20's commitment to ensuring all regions of the world are represented, and in consulting with countries beyond the G20 membership so as to understand fully their economic challenges, how they experience changes in the global economy and how G20 decisions affect them.

Each year, the G20's guests include Spain (a permanent invitee); the Chair of ASEAN; Global Governance Group; two African countries (the chair of the African Union and a representative of the New Partnership for Africa's Development (NEPAD)) and a country or countries invited by the presidency, usually from its own region. Since the first leader-level meeting of the G20 in 2008, guests have included Benin, Brunei, Cambodia, Chile, Colombia, Equatorial Guinea, Ethiopia, Kazakhstan, Malawi, the Netherlands, Spain, Switzerland, Thailand, the United Arab Emirates, Vietnam, Mauritania, Senegal, Myanmar and New Zealand.

In 2015 Turkey decided to invite Azerbaijan together with Malaysia, as the 2015 Chair of ASEAN; Senegal, representing NEPAD; Singapore, Spain and Zimbabwe, as the 2015 chair of the African Union.

\section{Otras aportaciones:}

The G20 committed in its 5th Anniversary Vision Statement to listen carefully to institutions and countries which are not in the group. This is because the G20's growth and resilience agenda matters to all countries, not just those in the G20. Growth in G20 countries, which constitute 85 per cent of the global economy, is essential to growth and development elsewhere. 
In 2015, the G20 is focusing on Low Income and Developing Countries (LIDCs) with a view to highlighting the relevance of G20's development agenda for these countries. 2015 offers a unique opportunity to enhance global development agenda. G20 has a strong role to play in that.

5. Las cumbres de Jefes de Estado y de Gobierno se celebran desde 2008:

14 y 15 de noviembre de 2008 en Washington, Estados Unidos.

2 de abril de 2009 en Londres, Reino Unido.

24 y 25 de septiembre de 2009 en Pittsburgh, Estados Unidos.

26 y 27 de junio de 2010 en Toronto, Canadá.

11 y 12 de noviembre de 2010 en Seúl, Corea del Sur.

3 y 4 de noviembre de 2011 en Cannes, Francia.

18 y 19 de junio de 2012 en Los Cabos, México.

5 y 6 de septiembre de 2013 en San Petersburgo, Rusia.

15 y 16 de noviembre de 2014 en Brisbane, Australia.

15 y 16 de noviembre de 2015 en Antalya, Turquía.

Nota de accesibilidad: Se puede solicitar el contenido de este estudio en Braille, a la dirección de correo electrónico <Enrique.Belda@uclm.es>

TITLE: G-20: Structure and fitness for its targets in international organizational and institutional framework

RESUMEN: El G 20 es un mecanismo instrumental, de escasa articulación institucional pero de amplísima capacidad de influencia, que con su definición de líneas de política económica y financiera agiliza la toma de decisiones por parte de países y organizaciones internacionales. Su impronta existencial pasa por procurar la apertura de la concertación mundial a las economías emergentes que se esconden tras Estados densamente poblados.

PALABRAS CLAVE: G 20; Instituciones económicas internacionales; Politica Exterior; Derecho Constitucional; Derecho Internacional Público.

ABSTRACT: The G20 is an instrumental mechanism, with limited institutional structuring, although with wide influence ability, with its definition of lines of economic and fiscal policy speeds up the decision making on the part of international countries and organizations. Its existential impression goes through obtain the global agreement opening to emerging economies hidden behind densely-populated countries.

KEY WORDS: G 20; International Economic institutions; Foreign policy; Constitutional law; Public international law. 